\title{
Caractérisation de voyelles en mélanges respiratoires synthétiques sous fortes pressions
}

\author{
M. GUITTON, J. CRESTEL et L. CHARONNAT \\ ENSSAT/LASTI, 6 rue de Kérampont, BP. 447, 22305 Lannion, France
}

\begin{abstract}
Résumé: Les plongeurs qui travaillent aux grandes profondeurs inhalent des mélanges respiratoires synthétiques. L'action conjuguée de la profondeur et des caractéristiques physiques des mélanges entraînent une perte d'intelligibilité quasi-totale de la parole produite. Cet article vise à mieux caractériser la déformation du signal de parole en conditions "hyperbares" avec pour objectif une restitution optimale de l'intelligibilité par traitement numérique du signal. L'étude proposée confronte l'évolution formantique de voyelles analysées à partir d'enregistrements réalisés au cours de la plongée expérimentale HYDRA X (COMEX, Nov 92) à l'évolution théorique résultant de l'application de la loi de Fant-Lindquist et de la modélisation n-tubes du conduit vocal. Les résultats obtenus pour les 3 voyelles $[a, e, i]$ sont présentés et discutés dans cet article.
\end{abstract}

\begin{abstract}
Divers working on deep submarine sites are breathing synthetic gas mixtures. The joint effects of depth and the physical features of the breathing mixtures produce significant loss of intelligibility. This paper aims to further the knowledge of speech alteration in order to restore the speech intelligibility with greater efficiency, by signal processing methods. This study compare the shift of natural vowels formant with the results of synthetic vowels computed from an $n$-tube model or from the Fant-Linquist's law. The "helium" speech was recorded during the experimental dive HYDRA X at COMEX (Marseille) at depths of up to 700 meters.
\end{abstract}

\section{INTRODUCTION}

Le modèle linéaire de production de parole est régi par la relation: $\mathrm{x}(\mathrm{t})=\mathrm{u}(\mathrm{t}) * \mathrm{~h}(\mathrm{t})$.

$\mathrm{h}(\mathrm{t})$ est la réponse impulsionnelle du filtre qui caractérise le comportement du conduit vocal en incluant l'effet des différents facteurs de pertes, des vibrations de parois et du rayonnement aux lèvres, $u(t)$ modélise le signal de source et le symbole $*$ représente l'opérateur de convolution.

Les modèles de source glottique et de conduit vocal doivent quantifier l'évolution du signal de parole lorsque les paramètres physiques des mélanges respiratoires varient: la masse volumique $\rho$, la célérité du son $\mathrm{c}$, la constante adiabatique $\eta$, le coefficient de conduction de chaleur $\lambda$ et la chaleur spécifique à pression constante $C_{p}$. Une étude précédente [1] a montré que l'incidence de la modification du comportement de la source vocale pouvait être négligée relativement à celle du conduit vocal; en conséquence un modèle paramétrique de source indépendant de la configuration articulatoire s'avère suffisant. Le modèle de référence de conduit vocal est le modèle n-tubes, les fonctions d'aire retenues pour la production des voyelles [a, e, i] sont extraites de l'étude de MRAYATI [2], les paramètres des mélanges respiratoires sont évalués pour les mélanges héliox et hydréliox de l'expérience HYDRA X de la Comex. 
La déformation spectrale du signal de parole résulte de la modification de l'ensemble des paramètres des mélanges, cependant la contribution de la célérité et de la masse volumique s'avère prépondérante [1]. L'étalement spectral est proportionnel à la célérité, la position relative des formants, conditionnée par le phénomène de vibrations de parois, est fonction de la masse volumique. Le dernier phénomène entraîne, pour la voyelle [i], un rapport de déplacement du premier formant supérieur à 6 aux grandes profondeurs ( $>300 \mathrm{~m}$ ) alors qu'il ne dépasse pas 3 pour les autres formants. Les valeurs de déplacement estimées par le modèle ne sont pas directement applicables en ce qui concerne les algorithmes de correction car elles sont dépendantes de la configuration articulatoire. La restauration des fréquences de formants exploite généralement la loi de Fant-Lindquist [3] :

$$
\mathrm{f}_{\mathrm{h}}^{2}=\left(\frac{\mathrm{c}_{\mathrm{h}}}{\mathrm{c}_{\mathrm{a}}} \mathrm{f}_{\mathrm{a}}\right)^{2}+\left(\frac{\rho_{\mathrm{h}}}{\rho_{\mathrm{h}}}-1\right)\left(\frac{\mathrm{c}_{\mathrm{h}}}{\mathrm{c}_{\mathrm{a}}} \mathrm{F}_{\mathrm{wa}}\right)^{2}
$$

Pour un formant "air" de fréquence $f_{a}$, les valeurs des rapports de célérités et de masses volumiques des mélanges relativement à l'air et la fréquence de résonance $\mathrm{Fw}_{\mathrm{a}}$ du conduit vocal fermé aux lèvres imposent la valeur du formant hyperbare $f_{h}$. La valeur de $F_{\text {wa }}$ est fixée à $180 \mathrm{~Hz}$.

\section{LES VOYELLES SYNTHETIQUES}

Le modèle électrique du conduit vocal en détermine la fonction de transfert $T(f)=U_{n+1} / U_{g}$ pour un nombre $\mathrm{n}$ de sections [2]. La fonction $\mathrm{T}(\mathrm{f})$ est discrétisée en fréquence sur l'intervalle [0..Fs], sachant que l'étalement spectral qui résulte de la modélisation et de la loi de FANT ne dépasse pas 3,2 les fréquences d'échantillonnage Fs sont fixées respectivement à $10 \mathrm{KHz}$ en milieu "air" et à $32 \mathrm{KHz}$ en milieu "hyperbare". La fonction discrétisée T(k) est calculée sur 256 points dans l'intervalle [0..Fs/2] puis elle est dotée d'une symétrie hermitienne, une transformation de Fourier inverse détermine la réponse impulsionnelle sur 512 points $[4,5]$. Pour la détermination précise des fréquences de formants la résolution est augmentée jusqu'à $1 \mathrm{~Hz}$.

L'onde glottique $\mathrm{g}(\mathrm{k})$ est définie pour les mêmes valeurs d'échantillonnage que la fonction de transfert. La forme de l'onde glottique est considérée invariante en fonction des paramètres physiques des mélanges. Statistiquement la période du pitch est inférieure en "hyperbare", cependant les échantillons traités dans l'étude expérimentale ont une période de pitch très proche d'une période de pitch "air". La durée du fondamental est fixée à $10 \mathrm{~ms}$ en "air" et en "hyperbare". L'onde de source est paramétrée pour l'air par:

\begin{tabular}{|c|c|c|c|}
\hline $\mathrm{Fe}=10 \mathrm{KHz}$ & $b=41$ & \multicolumn{2}{|c|}{$c=100$} \\
\hline$g(k)=0,5[1-\cos \pi \mathrm{k} / \mathrm{a}]$ & pour $0 \leq$ & k & $\leq \mathrm{a}$ \\
\hline $\begin{array}{l}g(k)=1-0,8[1+\cos \pi k / a] \\
g(k)=0\end{array}$ & $\begin{array}{l}\text { pour } a< \\
\text { pour } b<\end{array}$ & $\begin{array}{l}\mathrm{k} \\
\mathrm{k}\end{array}$ & $\begin{array}{l}\leq \mathrm{b} \\
<\mathrm{c}\end{array}$ \\
\hline
\end{tabular}

Les voyelles synthétiques résultent de la convolution de cette onde par la réponse impulsionnelle du filtre.

\section{RESULTATS}

Les résultats de l'analyse des voyelles synthétiques laissent apparaitre des fréquences de formants très proches des fréquences initiales des pôles de la fonction de transfert, dans ces conditions nous adopterons ces dernières comme référence.

Les voyelles réelles $[a, e, i]$ sont issues d'un corpus enregistré lors de l'expérience HYDRA X. La sélection des enregistrements (qualité, disponibilité à de nombreuses profondeurs, corpus suffisant) nous a conduit à ne retenir que des enregistrements issus d'un seul locuteur. Les 3 premiers formants de chacune des voyelles sont évalués dans les conditions suivantes: fréquence d'échantillonnage $32 \mathrm{KHz}$, analyse LPC 32 coefficients, préaccentuation de 0,95 , pondération de Hamming, fenêtres de 512 échantillons. De façon analogue à l'air les fréquences de formants de voyelles sont modifiées par les consonnes initiales et finales des phonèmes. Pour s'affranchir en partie de ce phénomène nous considérons la partie la plus stable de la voyelle pour différents phonèmes:

$\begin{array}{lllll}\text { voyelle } \mathbf{a} & \text { fAce } & \text { cAnard } & \text { Annie } & \text { bAt } \\ \text { voyelle } \mathbf{e} & \mathrm{jEté} & \mathrm{dE} & \text { pEtit } & \text { pEtite } \\ \text { voyelle } \mathbf{i} & \text { annIe } & \text { mIse } & \text { Immédiatement } & \text { petIte }\end{array}$



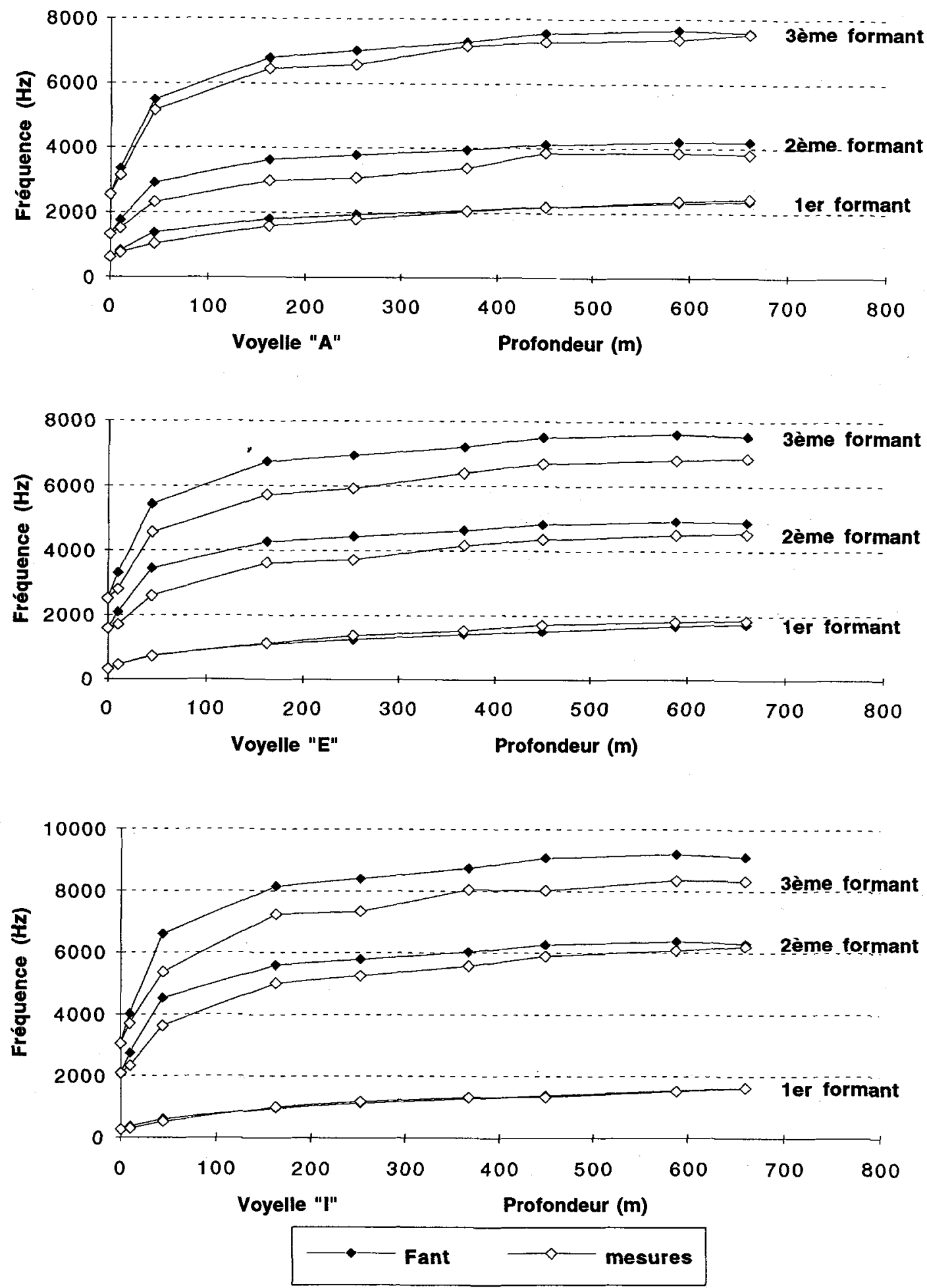

Figure 1 : Fréquences des 3 premiers formants des voyelles [a, e, i] 
Pour chacune des profondeurs retenues $(0,10,45,163,252,450,588,660 \mathrm{~m})$ et pour chaque voyelle nous calculons une valeur moyenne de la fréquence pour les formants F1, F2, F3, L'identification des formants devient plus difficile lorsque les profondeurs augmentent, dans certains cas un suivi de l'évolution de la fréquence du formant s'avère nécessaire. Les voyelles du locuteur ne sont pas disponibles dans l'air aussi les références "air" résultent d'une moyenne sur des échantillons des mêmes phonèmes pour plusieurs locuteurs.

Une évaluation préliminaire compare les valeurs de fréquence des formants issues de 2 types de modélisation: le modèle $\mathrm{n}$-tubes et la loi de Fant. La fréquence $\mathrm{F} 1$ du modèle est généralement supérieure à celle prévue par la loi de Fant (jusqu'à 10\%) sauf pour la voyelle [i] où les 2 valeurs restent proches (en moyenne <2\%). En ce qui concerne F2 et F3 les fréquences estimées par le modèle sont de 2 à $3 \%$ supérieures aux fréquences estimées par la loi de Fant.

Les résultats calculés par la loi de Fant, indépendants de la configuration articulatoire, ne prennent en compte que les 2 paramètres physiques essentiels des mélanges et cependant restent très comparables aux résultats issus d'une modélisation plus complexe. Dans la dernière phase les fréquences obtenues par l'analyse sont confrontées avec la loi de Fant (figure 1). Les écarts sur F1 s'avèrent faibles aux profondeurs importantes $(<5 \%)$ sauf pour la voyelle [e] qui dans quelques cas présente des écarts plus nets (jusqu'à $15 \%$ ). Les 2 èmes et 3èmes formants présentent des écarts nettement plus importants. Les fréquences obtenues par la loi de Fant sont surestimées d'un facteur de l'ordre de $10 \%$.

\section{CONCLUSION}

L'étude confirme globalement la validité du modèle n-tubes et de la loi de Fant pour les profondeurs maximales envisageables lors de plongées en saturation. Les résultats obtenus aux très grandes profondeurs prouvent que le comportement de l'appareil vocal reste satisfaisant, ce qui laisse envisager la possibilité d'algorithmes correcteurs efficaces. Les voyelles synthétiques "hyperbares", observées dans le domaine temporel, semblent relativement proches des voyelles réelles "hyperbares", ceci est moins vrai en ce qui concerne les voyelles "air". Une approche statistique fondée sur [6] va permettre de quantifier ces observations. La prochaine étape consiste à affiner les résultats en s'appuyant sur des références "air" plus fiables et en ajustant les paramètres des modèles.

\section{Références:}

Colloques: [1] J. Crestel, M. Guitton. Application de la modélisation de l'appareil phonatoire à la caractérisation de la parole hyperbare. Congrès Français d'acoustique Lyon, avril 1990.

Thèses: [2] M. Mrayati. Contributions aux études sur la production de la parole. Thèse d'état, Université de Grenoble, 1976.

Périodiques: [3] G. Fant, J.Linquist. Pressure and gas mixture effects on diver's speech. STQL-QPSR-1 pp. 7-31, Royal Inst. Techn. Sweden, 1968.

Colloques :[4] J. Crestel, M. Guitton, V. Le Calvé, M. Corazza. Sur la quasi-stationnarité du filtre vocal en conditions hyperbares, Colloque Gretsi, 1991, Juan-Les-Pins.

Colloques :[5] M. Guitton, J. Crestel. Characterization of vocal tract impulse responses for articulatory configurations of vowels in hyperbaric conditions, European Speech Communication Association Workshop, 1992, Cannes.

Colloques :[6] J. Crestel, M. Guitton. Estimation of speech signal classification features in a simulated hyperbaric environment, Eurospeech 1993, Berlin. 